\title{
ARTICLE
}

Received 23 Jul 2014 | Accepted 8 Oct 2014 | Published 19 Nov 2014 DOl: 10.1038/ncomms6501

\section{Atomically resolved tomography to directly inform simulations for structure-property relationships}

\author{
Michael P. Moody ${ }^{1}$, Anna V. Ceguerra², Andrew J. Breen², Xiang Yuan Cui ${ }^{2}$, Baptiste Gault',
} Leigh T. Stephenson ${ }^{2}$, Ross K.W. Marceau ${ }^{3}$, Rebecca C. Powles ${ }^{2} \&$ Simon P. Ringer ${ }^{2}$

Microscopy encompasses a wide variety of forms and scales. So too does the array of simulation techniques developed that correlate to and build upon microstructural information. Nevertheless, a true nexus between microscopy and atomistic simulations is lacking. Atom probe has emerged as a potential means of achieving this goal. Atom probe generates three-dimensional atomistic images in a format almost identical to many atomistic simulations. However, this data is imperfect, preventing input into computational algorithms to predict material properties. Here we describe a methodology to overcome these limitations, based on a hybrid data format, blending atom probe and predictive Monte Carlo simulations. We create atomically complete and lattice-bound models of material specimens. This hybrid data can then be used as direct input into density functional theory simulations to calculate local energetics and elastic properties. This research demonstrates the role that atom probe combined with theoretical approaches can play in modern materials engineering.

\footnotetext{
${ }^{1}$ Department of Materials, University of Oxford, Parks Road, Oxford OX1 3PH, UK. ${ }^{2}$ Australian Centre for Microscopy and Microanalysis, School of Aerospace, Mechanical and Mechatronic Engineering, The University of Sydney, Sydney, New South Wales 2006, Australia. ${ }^{3}$ Institute for Frontier Materials, Deakin University, Geelong, Victoria 3216, Australia. Correspondence and requests for materials should be addressed to M.P.M. (email: michael.moody@materials.ox.ac.uk).
} 
A tomic-scale microscopy enables the characterization of incredibly small and complex structures, and increasingly is the gateway for advances in materials technology. However, it is the complementary development of computational materials science that has been key to unlocking our understanding of the mechanisms by which atomic architectures directly impact the properties and behaviour of materials. Impressive inroads have been made in materials characterization over the last decade, particularly through continued advances in the capabilities of electron microscopy, most notably the efforts in high-resolution aberration-corrected transmission electron microscopy $(\mathrm{TEM})^{1}$ and scanning TEM $^{2}$ and the development of tomographic TEM techniques ${ }^{3}$. Atom probe tomography (APT) is a very different three-dimensional (3D) microscopy concept, but one that is rapidly rising in prominence ${ }^{4-6}$. On the basis of sequential field evaporation of individual ions from the surface of a sharp needle-shaped specimen, APT provides a highly accurate elementally resolved, atom-by-atom 3D image of a specimen encompassing hundreds of millions of atoms (Supplementary Fig. 1).

The data generated by APT is of a format almost identical to that exploited by many types of computer simulations. Indeed, one of the necessary precursors to carrying out atomistic modelling is to have the initial microstructure configurations as close as possible to real structures ${ }^{7}$. In constitutive modelling of inhomogeneous systems, grid points or supercells are often employed to represent different parts of the microstructures. A fundamental problem with such an approach is that the actual microstructures may differ significantly from simulated ones because of the presence of non-uniform distributions of crystal orientation, and morphology . $^{8}$

APT has previously been used to bridge this gap between experimental microscopy and atomistic simulation. Many studies have demonstrated this potential by using atom probe data to indirectly inform various flavours of computational materials science, including Monte Carlo (MC) simulations ${ }^{9-12}$, molecular dynamics $(\mathrm{MD})^{13}$ and density functional theory (DFT) ${ }^{14-19}$. In each case, APT offers valuable information to construct relevant models, and the simulated results can subsequently be compared with experiments. However, although APT reconstructions represent a highly spatially resolved atomistic representation of a material system, this data remains both imperfect and significantly incomplete. This is key since it prevents APT data from being seeded directly into theoretical models and simulations, and thus prevents the full potential of its information from being realised.

The 3D spatial resolution of APT can be extremely high ${ }^{20,21}$, however, the location of atoms in the reconstructed image is not exact. As a result, in the analysis of crystalline materials, atoms are offset from their true positions on the lattice. Further, due to the incorporation of multi-channel plates in the design of the position-sensitive detectors, a significant proportion of ions striking the detector are simply omitted from the final reconstructed image. This process is often considered stochastic and independent of the identity of the incident ion. The term detection efficiency refers to the fraction of ions that are successfully detected, and in most commercial instruments has an estimated value of $\varepsilon \approx 0.37-0.57$. Imperfect spatial resolution and detection efficiency are two of the most serious issues limiting APT from offering true atomic resolution analyses. Instrumentation advances will continue to incrementally improve the situation, but this goal will not be achieved in the near future via technical advances alone.

In the present study, we propose a new analytical approach that blends experiment with simulation to create a complete and lattice-based atomistic image of the original system. Although the information provided by APT is incomplete, in actuality, the sheer amount of data generated by this technique is its exceptional strength. Millions of atoms are routinely chemically identified and precisely located in $3 \mathrm{D}$ in the course of an experiment. Hence, an almost overwhelming amount of statistical information is available and this is the foundation on which we have built our methods.

Our strategic approach is outlined step-by-step in Fig. 1. A crystalline specimen is analysed via APT (Fig. 1a), generating a $3 \mathrm{D}$ atomic reconstruction of the original atomic configuration (Fig. 1b). In the next step, the average local chemical distribution within the reconstruction is characterized by measuring a set of short-range order (SRO) parameters at increasingly large radii (Fig. 1c i-iv). SRO parameters are well established for the characterization of chemical correlations in alloyed materials, and we have recently developed an approach to derive these measurements from APT data ${ }^{22}$. It was also demonstrated that SRO measured via APT can provide a quantitative description of the nanostructure present in the system despite its aforementioned limitations.

On further statistical analyses, in many cases, it is observed that significant crystallographic information has been retained in the reconstruction ${ }^{23-25}$ (Supplementary Fig. 2). This is discussed in more detail in Supplementary Note 1. This information can be used to correct the data, atom by atom, to the perfect lattice configuration of the original specimen ${ }^{26,27}$ (Fig. 1d). We term this process lattice rectification. In the rectified data, it will be apparent that there are a substantial number of vacant lattice sites. As a first approximation, it is assumed that this is due solely to a limited detection efficiency, which, in a stochastic manner, effectively omits a significant fraction of the ions field evaporated from the specimen. Atoms can then be replaced into the APT data by filling the vacant sites, and these will be termed restored atoms. The chemical identity of individual restored atoms is chosen at random but with the constraint that after all vacant lattice sites have been filled, the overall composition of the original reconstruction has been maintained (Fig. 1e).

It is clear from comparing the respective chemical distributions in Fig. 1d,e that most of the restored solute atoms have been placed in incorrect lattice sites. This is also reflected in a drastic change in values when the SRO parameters are re-measured because we are randomly adding atoms to a reconstruction that has significant short-range ordering. At this point, the reconstruction is seeded directly into to a MC algorithm. This MC simulation is specifically adapted such that instead of utilizing interaction potentials, trial moves are accepted or rejected based on their effect on the overall SRO of the system. The SRO parameters measured in the original reconstruction (Fig. 1c) are used by the simulation as target values. Atomic positions continue to be swapped at random until the SRO target values have been restored ${ }^{28}$. A critical constraint applied to this simulation is that the positions of the original (experimentally detected) atoms are not moved from their lattice-rectified positions, only the positions of the restored atoms are swapped. The final result is a $3 \mathrm{D}$ lattice-based and atomically complete representation of the nanostructure present in the original experimental specimen (Fig. 1f).

\section{Results}

Replacing atoms missing in simulated systems. There is no way of confirming the chemical identities of the actual ions lost in the experiment due to detection efficiency; hence, computer-generated APT-like systems provide an excellent means to test the accuracy of our approach for predicting the missing atoms. A range of systems were simulated, each incorporating its own 
a
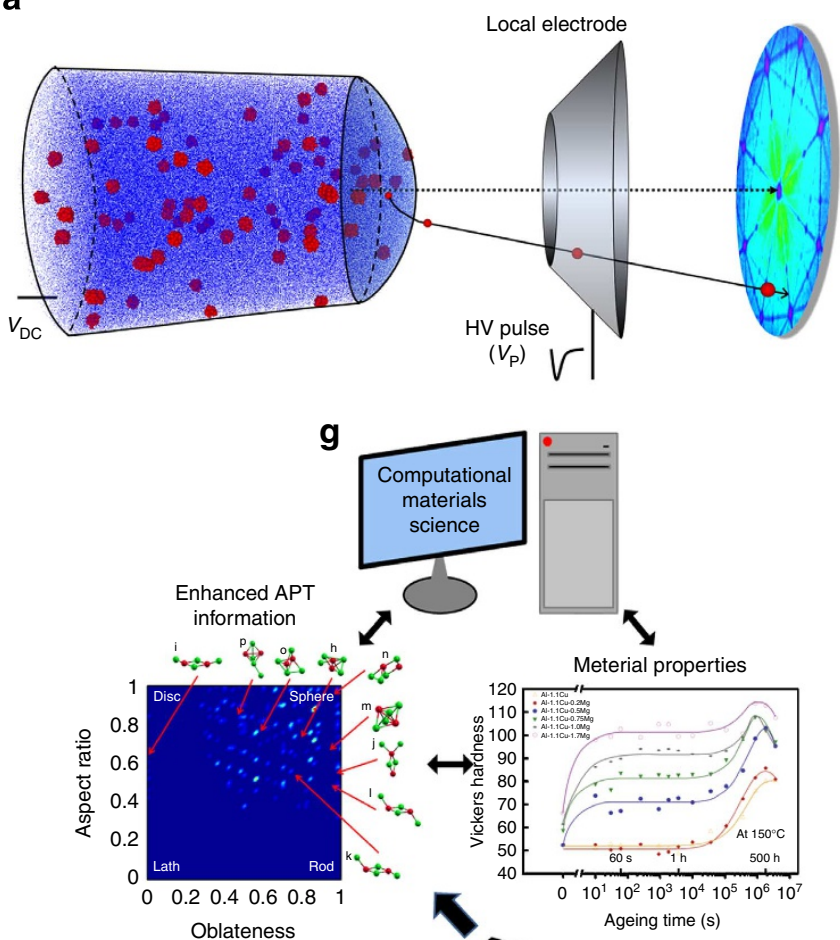

Oblateness

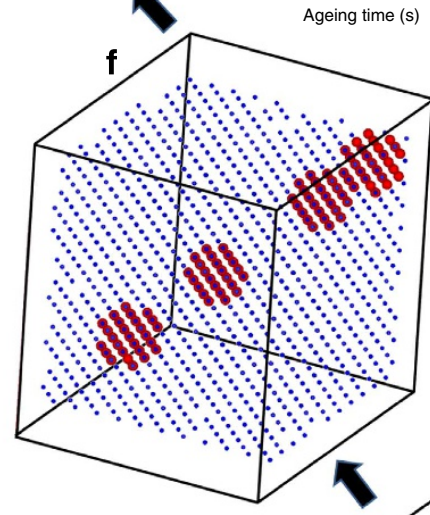

(1) b

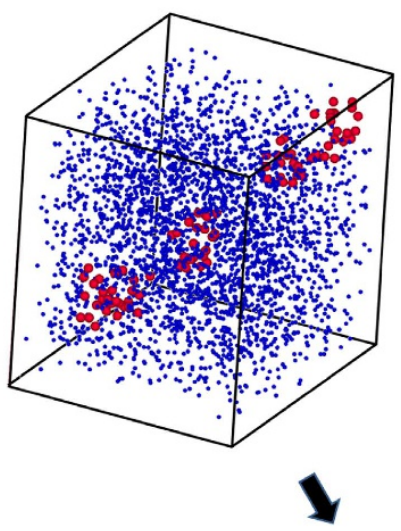

C
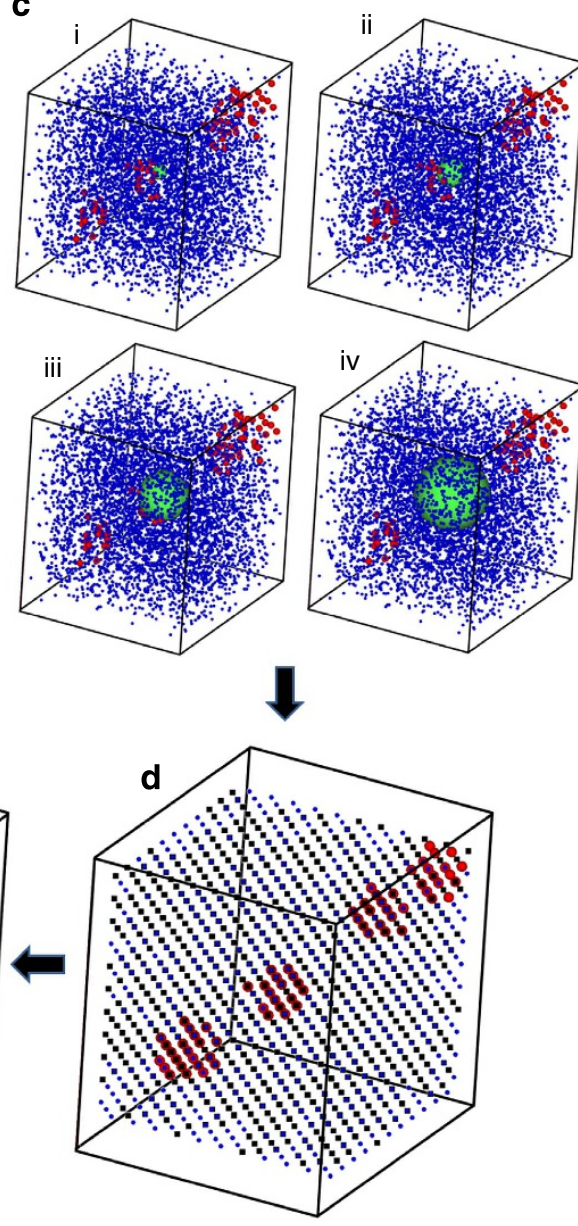
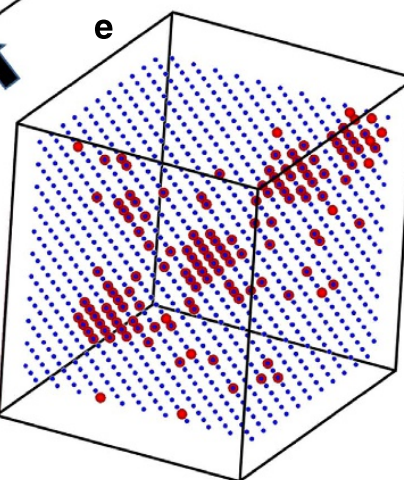

Figure 1 | Approach for corrected and completed atom probe data. (a) Schematic of atom probe experiment. (b) Close-up of a very small subvolume of APT reconstruction. Small blue spheres represent matrix atoms and large red spheres are solute atoms. (c) SRO is measured by characterization of the local neighbourhood surrounding each atom for increasingly large radii (i-iv). (d) Reconstruction is lattice rectified. The positions of vacant lattice sites due to limited detection efficiency in the experiment are highlighted by black squares. (e) Atoms are replaced at random into the vacant sites while maintaining the original bulk composition. (f) SRO measured in $\mathbf{c}$ is re-established by swapping positions of restored atoms in a MC simulation. (g) Resulting data informs computational materials science.

unique nanostructure. In the first case, a series of three ternary $(A-B-C)$ systems was simulated on a face-centred cubic lattice. In these simulations, the $B$-type solute atoms occurred exclusively in spherical precipitates, while the $C$-type solutes occurred randomly throughout the matrix. An example of these simulations is presented in Fig. 2a. The effect of randomly removing $63 \%$ of atoms from the system, to model the effects of detection efficiency in a reflectron-fitted atom probe, is demonstrated in Fig. 2b. The generalized-multicomponent SRO (GM-SRO) parameters were then measured by characterizing the chemical distribution within each of the first five crystallographic shells around every atom ${ }^{22}$. The now-vacant sites on the lattice represent the positions of undetected atoms. Atoms with randomly chosen chemical identities were then restored to these vacant sites as shown in 
a

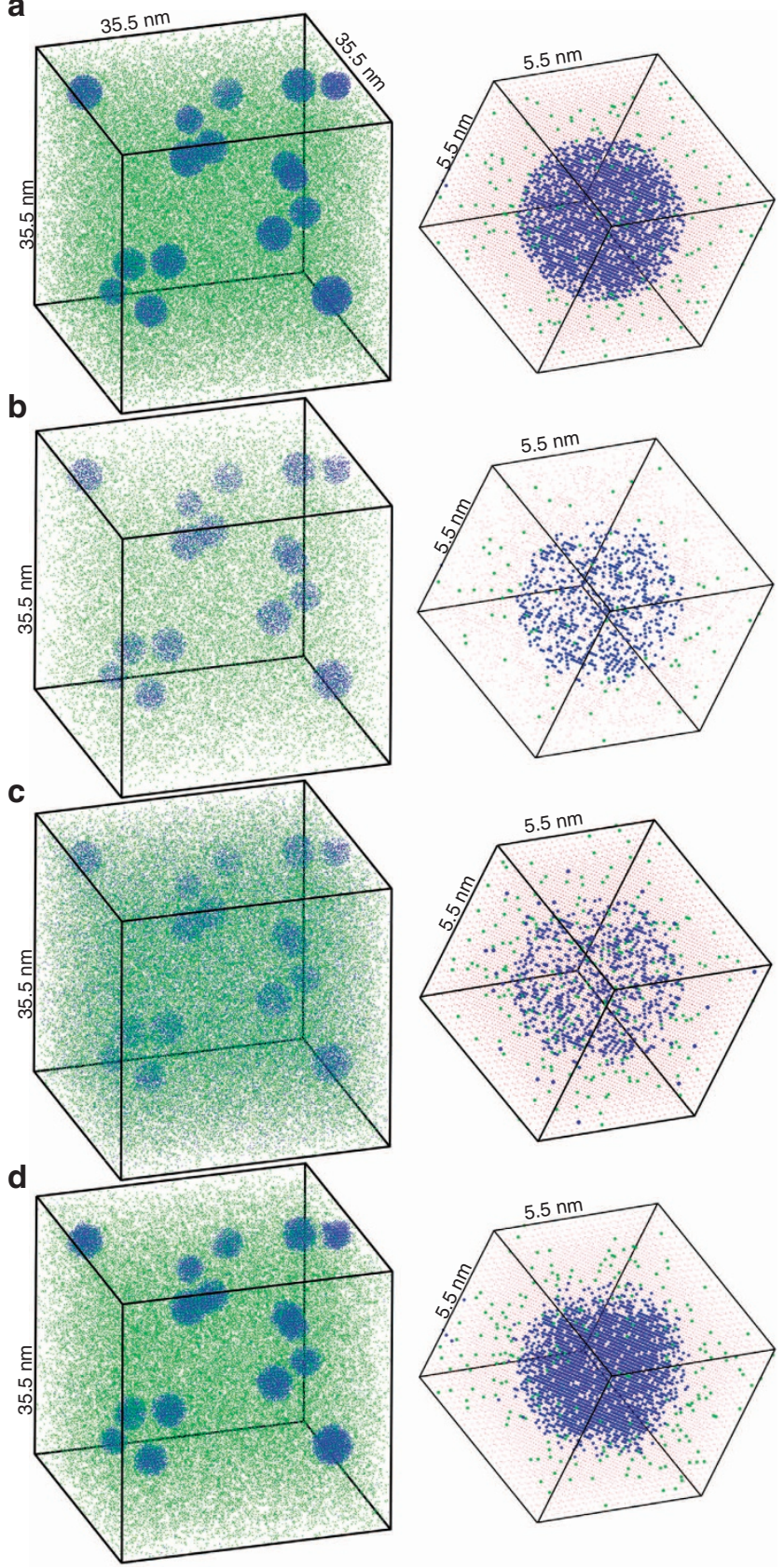

Figure 2 | Restoring atoms to simulated system. (a) On left is the original simulation (B-type atoms are blue dots, C-type atoms are green and matrix atoms are not shown at this scale for sake of visual clarity). On right is a close-up of a specific precipitate. Matrix atoms are now shown and are now depicted by red dots. (b) Same simulation after $63 \%$ of atoms have been removed at random. On right is corresponding close-up of same precipitate. (c) Atoms are replaced at random in the vacant lattice sites. The overall composition of the system has been maintained. (d) The final system after distribution of the restored atoms is rearranged via $M C$ simulation to return the original SRO.

Fig. 2c, while maintaining the overall composition of the system. The data were then seeded directly into the MC simulation, and the originally measured values of the GM-SRO parameters were re-established by swapping the positions of the restored atoms. The final result is presented in Fig. 2d, in which overwhelmingly the solute atoms have been returned correctly to the precipitates.
Supplementary Note 2 describe how the approach was also successfully applied to systems containing more complicated nanostructures, including precipitates with an ordered structure (for example, $\mathrm{L1}_{2}$ in Supplementary Fig. 3) and features with non-spherical geometries (for example, discshaped Guinier-Preston zones in Supplementary Fig. 4). The results demonstrate that based on the existing, but incomplete data, not only were atoms accurately replaced back into a precipitate, but also the existing long-range order and shape of these nanostructural features were preserved. It emphasizes the fact that the different crystallographic shells surrounding each atom inherently contain information about the site occupation of elements along different crystallographic orientations.

Application to experimental data. This approach was then applied to replace the missing atoms in actual experimental APT data. A thermally treated $\mathrm{Al}-5.6 \mathrm{Ag}-0.84 \mathrm{Cu}$ (at. \%) alloy analysis was chosen for this investigation since it contains a significant amount of nanostructure in the form of spherical, coherent $\mathrm{Ag}$ Guinier-Preston zones (Fig. 3a; Supplementary Fig. 1). The data were obtained on a straight-flight path local electrode atom probe 3000X Si. Multiple sets of crystallographic planes in the data were identified and characterized using spatial distribution maps ${ }^{29}$ (Supplementary Fig. 2). The precision of both the SRO measurement and the lattice rectification procedure is dependent on the spatial resolution of the APT data to which they are applied. Hence, a $20 \times 20 \times 100 \mathrm{~nm}$ region of interest (ROI), containing $1.2 \times 10^{6}$ atoms, free of significant reconstruction artefacts was then identified and isolated. These data are shown in Fig. 3a where at this scale only solute atoms are shown. A close-up within this ROI is also presented in which all the atoms (matrix and solute) are shown.

Next, the complete set of GM-SRO parameters, $\left[\alpha_{\mathrm{Al}-\mathrm{Al}}, \alpha_{\mathrm{Al}-\mathrm{Ag}}\right.$, $\left.\alpha_{\mathrm{Al}-\mathrm{Cu}}, \alpha_{\mathrm{Ag}-\mathrm{Al}}, \ldots, \alpha_{\mathrm{AlAgCu}-\mathrm{AlCu}}, \alpha_{\mathrm{AlAgCu}-\mathrm{AgCu}}\right]$, was measured for the first five crystallographic shells where $\alpha_{\mathrm{AB}-\mathrm{CD}}$ represents the distribution of $C$ - and $D$-type atoms around $A$ - and $B$-type atoms. A key assumption that we make is that ion detection is effectively a stochastic process, and hence, although a significant fraction of atoms are missing from the final reconstruction, this does not affect the values of the measured SRO, that is, the SRO we measure from a data set with $57 \%$ detection efficiency is the same as that which would be measured in a hypothetical $100 \%$ detection efficiency data set. The validity of this assumption has been previously investigated ${ }^{28}$. It is also well known that in certain analyses can occur a non-stochastic loss of ions due to a combination of preferential evaporation and detector pile-up effects $^{30,31}$, however, this is not expected to be a significant issue for the alloys chosen for this study.

Following this, the ROI was subject to lattice rectification. It has previously been estimated that in a reasonable-sized subvolume of a pure $\mathrm{Al}$ reconstruction upwards of $85 \%$ of the rectified atoms is returned to their correct lattice sites ${ }^{26}$. The effect of restoring the lattice is clearly apparent when comparing the close-ups in Fig. 3a,b. The vacant lattice sites were then identified and their positions are highlighted in Fig. 3b by black squares. As for the simulations described above, atoms were restored to these vacant sites, their chemical identities chosen at random but with the constraint that the overall bulk composition remained the same throughout this process. A close-up of the restored atoms is shown in Fig. 3c. The corresponding image of the entire subvolume indicates the homogeneous distribution of the restored solute atoms throughout the system, and is in clear contrast to the distribution of the experimentally detected solutes in Fig. 3a. 
a

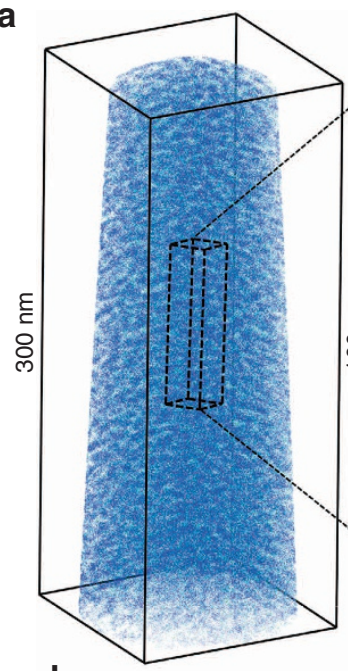

d

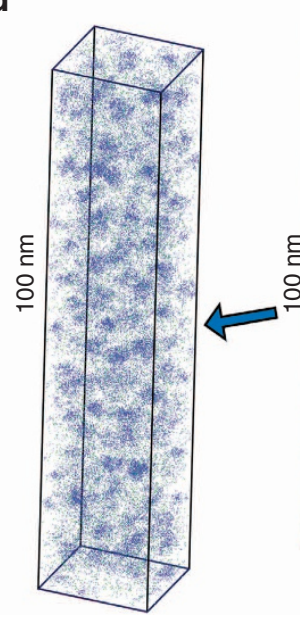

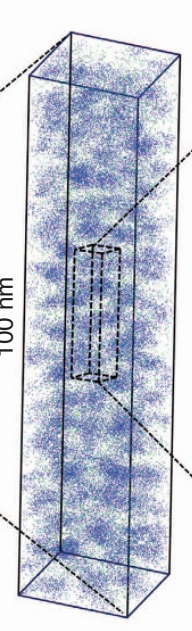
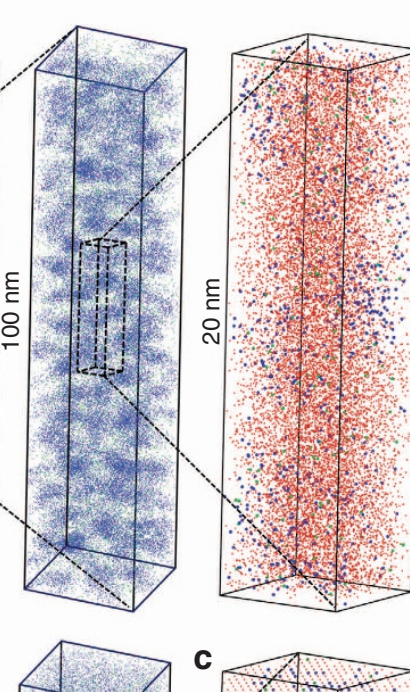

\section{$\checkmark$}

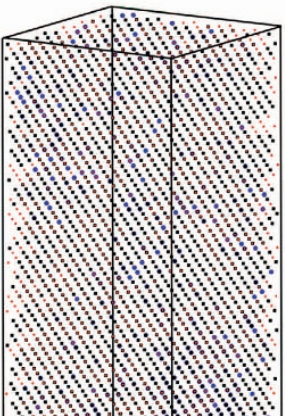

$\frac{1}{2}$

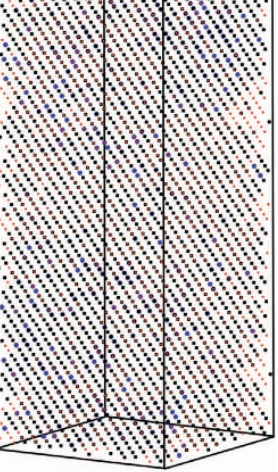

Figure 3 | Restoring missing atoms to actual APT data. (a) Left, Al-5.6Ag-0.84Cu (at. \%) APT reconstruction. ROI is highlighted, which contains $1.2 \times 106$ atoms. Only solute atoms (Ag blue, Cu green) are shown for clarity. Close-up within ROI also indicates position of matrix ions (Al red). (b) Closeup of ROI after lattice rectification, restoring face-centred cubic crystal. Vacant lattice sites are highlighted by black squares. (c) Close-up of positions of atoms randomly restored atoms to vacant sites. Corresponding image of entire ROI indicates the homogenous distribution of the restored solute atoms. (d) ROI distribution of the restored atoms after the MC simulation to restore SRO.

The entire data set (including both detected and restored atoms) was seeded into the MC simulation with target SRO based on the measured GM-SRO parameters that measured the original APT data. The final solute distribution of the restored atoms is presented in Fig. 3d. There is a striking agreement between the distribution of the detected and restored solute atoms. This is as expected, since they effectively represent two halves of the same material system that has been randomly split through the action of the microchannel plate detector system. Supplementary Fig. 5 shows a further example where this technique has been equally effective in restoring APT data containing precipitates with more complicated shapes. The final data set is a hybrid of experimental and simulated data, an approximation of a completed 3D image of the original specimen in which every atom is accounted for chemically and spatially.

APT-informed DFT calculation. There are many ways that the atomically complete hybrid APT data can potentially be used to inform simulations. The simplest example is a direct comparison between the nature and distribution of nanostructure characterized in the completed experimental data and that predicted by simulation. Alternatively, the hybrid APT data set could potentially actually be directly seeded into a kinetic MC or MD simulation to predict how the system will evolve with time, when subject to specific treatments or conditions. Here we implement DFT simulations to demonstrate local area energy mapping in the thermally treated $\mathrm{Al}-5.6 \mathrm{Ag}-0.84 \mathrm{Cu}$ (at. \%) system presented in Fig. 3. DFT simulation was chosen because it can in principle predict energetic, electronic and elastic properties of a specific atomic configuration. It is easy to envisage that local compositional variation will impact local properties, and then the macroproperties. However, precise quantification of realistic structures to undertake such a calculation is a grand challenge-hindering a true material optimization at the atomic level. To demonstrate this concept, we have carried out DFT simulations to calculate 'local cohesive energy', 'local Fermi energy' and 'local elastic moduli' within regions of the specimen. The cohesive energy is closely correlated with many properties, including interatomic bonding strength, molar volume and compressibility ${ }^{32}$, while the Fermi energy is critically important for the electrical and thermal properties of solids ${ }^{33}$.

To conduct the energy mapping, first, 49 groups of 'cubic' 32atom $(2 \times 2 \times 2$ supercells $)$ subvolumes were randomly sampled from the hybrid APT data, as illustrated in Fig. 4a. Then, the duplicity of these subvolumes was assessed, indicating 15 pure $\mathrm{Al}$ 
a

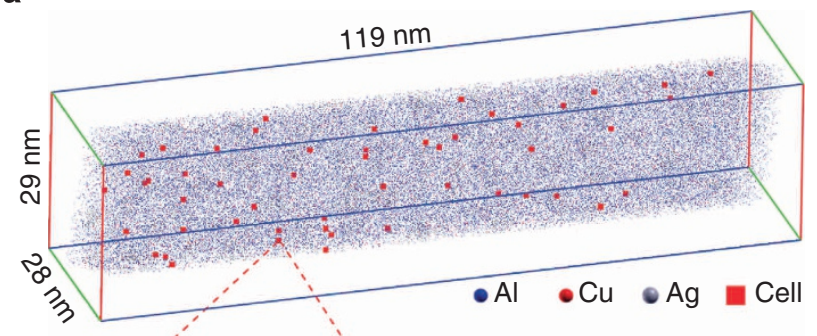

b

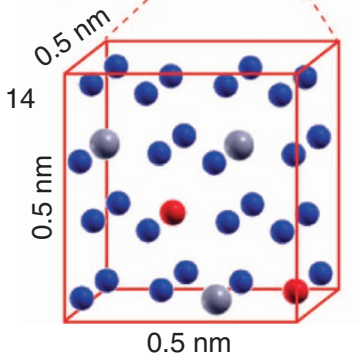

C

32-Atom local region

d

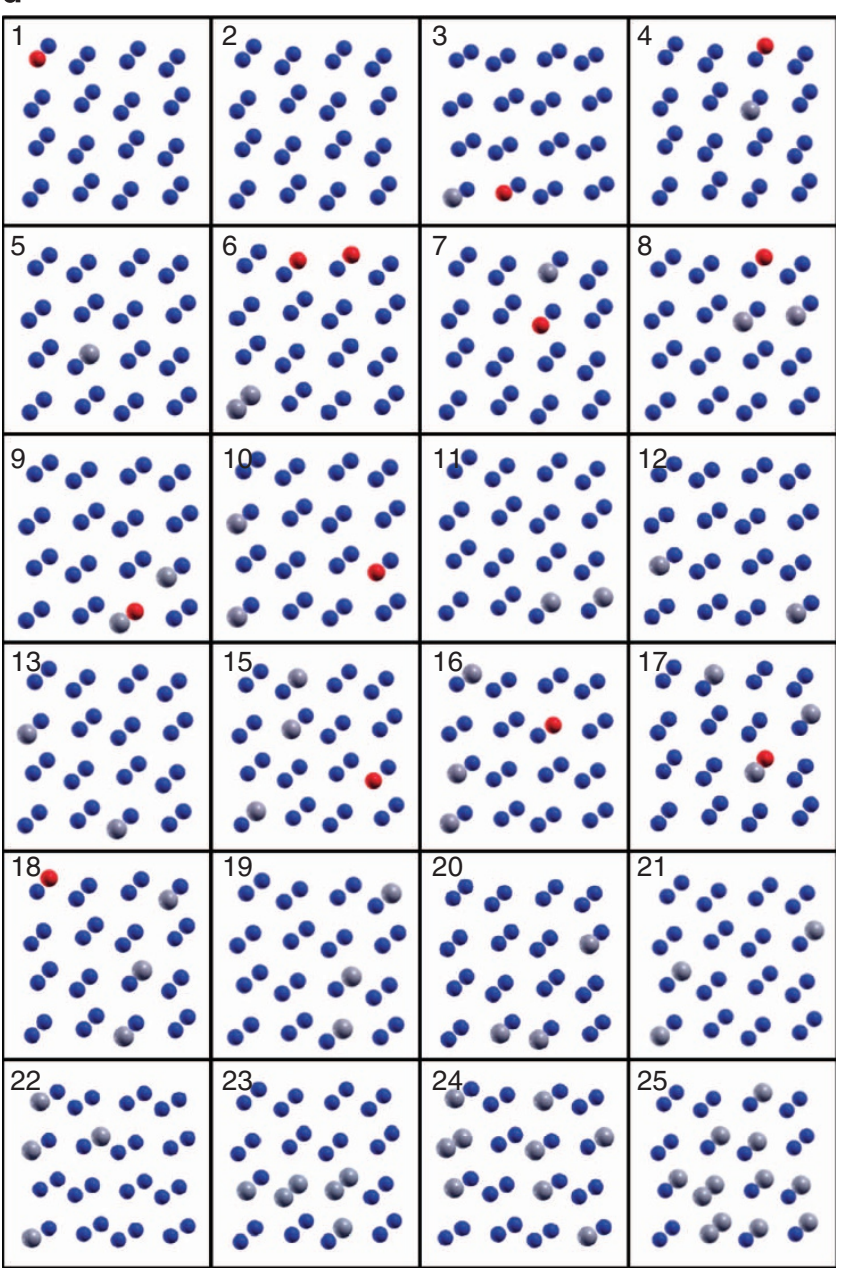

Figure 4 | Sampling regions of the hybrid APT data for local property

mapping. (a) Slice of the hybrid APT data, red squares indicate regions that have been randomly sampled. (b) Close-up of one of the sampled 32-atom subvolumes. (c) The subvolume in $\mathbf{b}$ embedded into a 108-atom supercell. (d) The unique 32-atom configurations. The sequence in which these configurations appear in this figure is based on the associated increase in cohesive energy calculated in Fig. 5. subvolumes and 8 subvolumes containing only a single Ag atom. In total, 25 unique configurations were identified, and these are presented in Fig. 4. To account for environmental effects and enable periodic boundary conditions, each unique 32 -atom configuration was then embedded into a 108-atom $3 \times 3 \times 3$ supercell. DFT total energy calculations were subsequently performed, allowing atomic relaxation, that is, possible structural distortions were taken into account.

The calculated local cohesive energy as a function of number of $\mathrm{Ag}$ atoms contained in each subvolume is presented in Fig. 5a. Local cohesive energy clearly decreases with a linear dependence on increasing number of local Ag atoms (goodness of fit of linear regression $\left.r^{2}=0.98\right)$. The $\sim 0.1 \mathrm{eV}$ per atom variation of cohesive energy across the Ag concentration range considered here is relatively small (that is, within the error range for standard DFT calculation for a given system). However, our systematic study consistently consolidates the reliability of this trend. Indeed, by examining two higher-concentration configurations, 32-Ag-atom and 108-Ag-atom, it has been confirmed that this linearity is still valid. Such behaviour is reasonable if one compares the cohesive energy values of bulk $\mathrm{Al}$ and $\mathrm{Ag}$. Experimental values are $3.39 \mathrm{eV}$ per atom for pure $\mathrm{Al}$ versus $2.95 \mathrm{eV}$ per atom for pure $\mathrm{Ag}^{34}$ and the corresponding values calculated in this study are $3.33 \mathrm{eV}$ per atom and $2.56 \mathrm{eV}$ per atom, respectively. In the calculations performed here, for a single $\mathrm{Al}$ and $\mathrm{Ag}$ atom, the spin polarisation and Hund's effects were explicitly included. It is also notable that, for each $\mathrm{Ag}$ concentration, increasing the number of $\mathrm{Cu}$ atoms tends to increase the local cohesive energy, indicating an attractive interaction between $\mathrm{Ag}-\mathrm{Cu}$ atoms.

The calculated local Fermi energy as a function of number of $\mathrm{Ag}$ atom is presented in Fig. 5b. Again, with increasing the Ag concentration, the Fermi energy decreases. The graph demonstrates that the local addition of $\mathrm{Cu}$ atoms further decreases the local Fermi energy (goodness of fit of linear regression $r^{2}=0.90$ ). This behaviour is to be expected since the Fermi energies of Ag and $\mathrm{Cu}$ are much smaller than that of $\mathrm{Al}$. Experimental values are $5.49 \mathrm{eV}$ for $\mathrm{Ag}, 7.00 \mathrm{eV}$ for $\mathrm{Cu}$ and $11.7 \mathrm{eV}$ for $\mathrm{Al}$ (ref. 33). The results demonstrate the importance of characterizing and controlling the local microstructure to tailor the micro- and ultimately macro-properties accordingly.

The final DFT example presented in this study uses the information in the unique hybrid APT data format to investigate the local mechanical and thermodynamic properties. In this case, the central parameters, namely, the elastic constants, are derived by applying a set of homogeneous deformations and calculating from the second derivative of the total energy as a function of volume, tetragonal and trigonal lattice distortions around the equilibrium structures. To our knowledge, a systematic comparison of elastic quantities as a function of local microstructure variation has not been previously reported. Due to the low symmetries involved, we have selected seven of the 32-atom configurations in Fig. 4, whose relaxed equilibrium cells are tetragonal (or cubic) structures. For these cases, expressions can be derived for bulk modulus,

$$
B=\frac{C_{33}\left(C_{11}+C_{12}\right)-2 C_{13}^{2}}{C_{11}+C_{12}+2 C_{33}-4 C_{13}},
$$

shear modulus

$$
G=\frac{C_{11}-C_{12}}{2}
$$

and Young's modulus,

$$
Y=\frac{9 B}{1+3 B / G}
$$




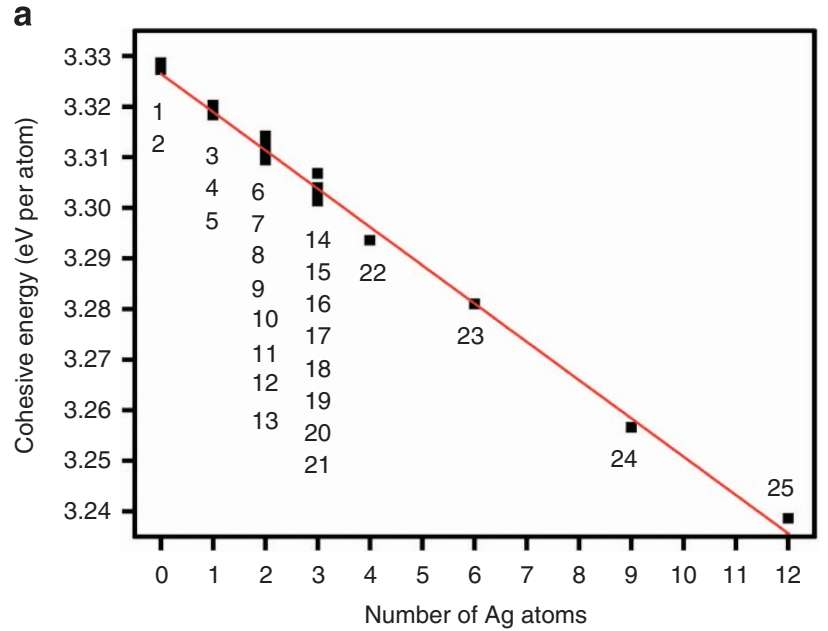

b

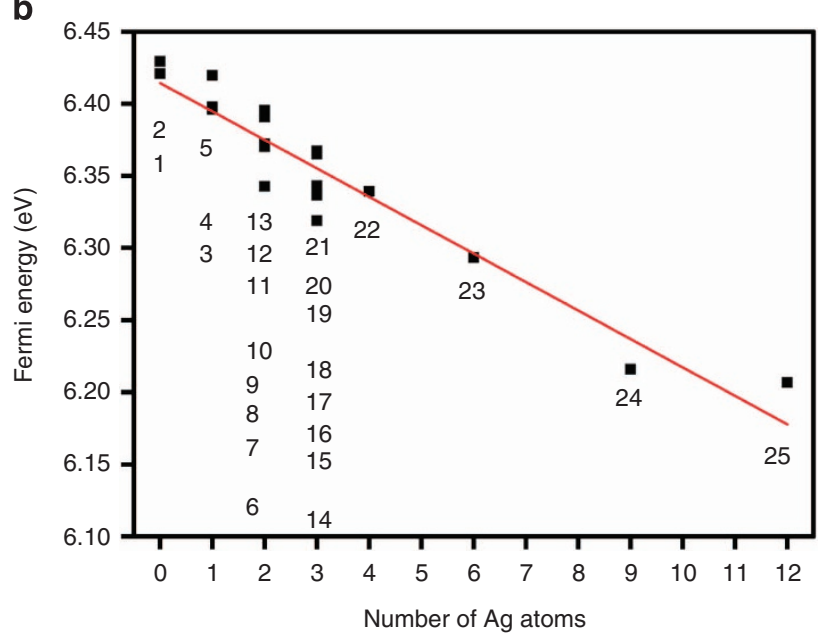

Figure 5 | DFT calculation of local energies as a function of Ag content. (a) Local cohesive energy and (b) local Fermi energy calculated for each unique 32-atom configuration.

\begin{tabular}{|c|c|c|c|c|c|c|}
\hline Configuration & $\begin{array}{c}\text { C11 } \\
(\mathrm{GPa})\end{array}$ & $\begin{array}{c}\mathrm{C} 12 \\
(\mathrm{GPa})\end{array}$ & $\begin{array}{c}\text { C13 } \\
(\mathrm{GPa})\end{array}$ & $\begin{array}{c}\text { C33 } \\
\text { (GPa) }\end{array}$ & $\begin{array}{c}\text { C44 } \\
(\mathrm{GPa})\end{array}$ & $\begin{array}{c}\mathrm{C} 66 \\
(\mathrm{GPa}) \\
\end{array}$ \\
\hline 1 & 98.2 & 56.4 & 56.4 & 98.2 & 26.7 & 26.7 \\
\hline 2 & 103 & 62 & 62 & 103 & 27.6 & 27.6 \\
\hline 3 & 110.7 & 66.2 & 64.4 & 110.2 & 29.7 & 31.9 \\
\hline 5 & 102.8 & 58 & 58 & 102.8 & 28.9 & 28.9 \\
\hline 7 & 110.7 & 64.4 & 66.2 & 110.7 & 29.8 & 30.2 \\
\hline 11 & 114.5 & 65.7 & 65.7 & 114.4 & 33.6 & 31.4 \\
\hline 19 & 116.1 & 65.1 & 66.2 & 117.1 & 29.5 & 28.6 \\
\hline
\end{tabular}

The values are sampled from within hybrid APT data (Fig. 4).

Values for the six independent elastic constants have been calculated and are listed in Table 1 . The resulting measurements of local elastic quantities are presented in Fig. 6, again plotted as a function of the number of Ag atoms.

First, from Table 1 it is apparent that all calculated elastic constants satisfy the criteria of mechanical stability for tetragonal crystals $^{35}: C_{11}>0, C_{33}>0, C_{44}>0, C_{66}>0,\left(C_{11}-C_{12}\right)>0,\left(C_{11}\right.$ $\left.+C_{33}-2 C_{13}\right)>0$ and $\left[2\left(C_{11}+C_{12}\right)+C_{33}+4 C_{13}\right]>0$. Hence, it

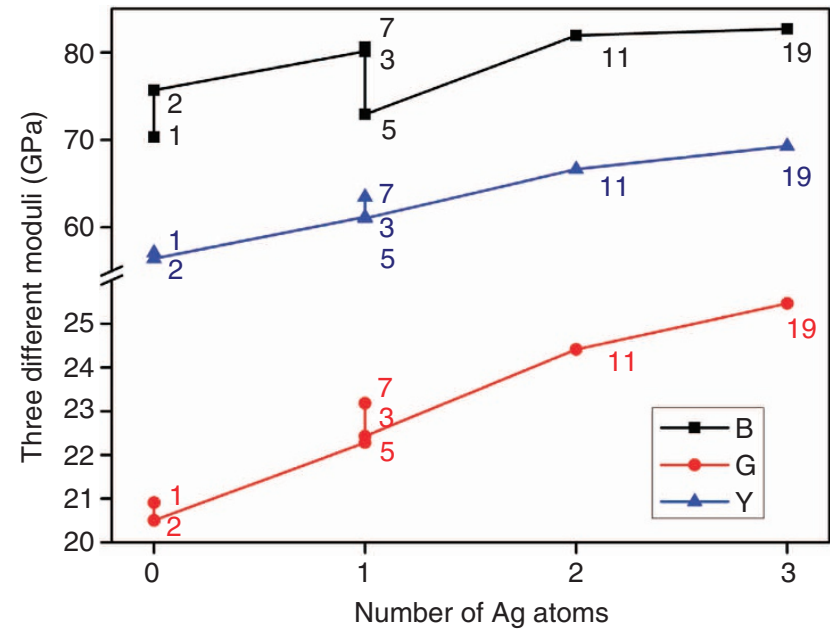

Figure 6 | Local bulk modulus (B), shear modulus (G) and Young's modulus (Y). Moduli calculated by DFT simulations informed directly by local regions of the hybrid APT reconstruction and plotted as a function of $\mathrm{Ag}$ content.

can be asserted that all the examined local configurations are intrinsically stable. Further, the general trend for each of these three moduli ( $B, G$ and $Y$ ) is similar, increasing with $\mathrm{Ag}$ concentration. Yet, for a given Ag concentration, the calculated order of moduli values varies. All the three moduli $(B, G$ and $Y$ ) can be used to evaluate material hardness, however, $G$ and $Y$ are better indicators ${ }^{36}$. The calculated $B, G$ and $Y$ values $(75.67,20.51$ and $56.42 \mathrm{GPa}$, respectively) for pure $\mathrm{Al}$ agree reasonably well with those derived experimentally $(76,26$ and $70 \mathrm{GPa})$. The calculations presented in Fig. 6 results consistently suggest that adding Ag will increase the local hardness.

\section{Discussion}

Microscopy has continued to make great strides in characterizing material microstructure with ever increasing details. However, there still remains an intrinsic disconnect between the microstructure and the bulk material properties. The way in which local microstructure variations influence the macro-property is poorly understood, restricting the potential to use information generated by microscopy for the purpose of materials' design. Hence, the aim of this study is twofold: first, from a microscopy perspective, the goal has been to extend the current limits of APT by generating complete hybrid experimental-analytical data sets of a form that can be seeded directly into atomistic simulations. Second, from the perspective of physical material properties, by using these hybrid data sets, we attempt to bridge the gap between microstructure and macro-properties by studying the local properties. The significance of this is that collectively these local properties will contribute to define to the macroscopic properties. For inhomogeneous systems, in many cases, the ability to accurately probe the local properties is highly desirable and represents a crucial step to understand the normally complicated bulk properties and to offer information for material design ${ }^{37,38}$. The results demonstrate the importance of characterizing and controlling the local microstructure to tailor micro- and ultimately macro-properties accordingly.

It must be noted that the method that has been developed to restore the data missing from APT characterization is based on a statistical process. The exact configuration of solute atoms that converges to a particular set of SRO parameters is not necessarily unique. Hence, the hybrid APT data set that is ultimately 
generated is one of the number of possible atomic configurations that could be obtained. However, this is analogous to examining two or more successive APT analyses of the same specimen (for example, a solid solution). Although the individual distributions of solutes will be statistically equivalent, at the atomic scale, identical configurations would never be observed. Hence, the hybrid APT data represents the most likely configuration of atoms based on the existing experimental information, which, although not unique, is a highly accurate representation of the true atomic structure of the material.

The approach also makes several key assumptions and approximations. These are acknowledged here to map out the challenges for routine and meaningful rectification and restoration of APT data into the future: it is assumed that detection efficiency is independent of ionic identity; data must be free of aberrant regions of high and low density; and the SRO analysis must have sufficient statistics to ensure that measurements are not unduly biased and are representative of the bulk system. Currently, all atoms are mapped back onto a perfect single crystal lattice by the rectification process. Hence, the alloy systems studied in this work were either solid solutions of engineering significance or alloy systems where precipitates are coherent with the surrounding matrix. The understanding gained from relatively simple systems is instrumental in defining the pathways to develop the infrastructure to investigate complex systems containing, for instance, interstitial atoms, defects, secondary phases or even grain boundaries. We envisage that this will be a future development of the methodology, which will require development of secondary corrections based on all of the available information. Most of the limitations listed above are issues affecting all APT-based characterizations, not just those developed in this study. As such, research is already ongoing towards limiting the influence of all of these effects. Indeed, instrumentation will undoubtedly continue to improve and rather than being relegated by such advances, the analytical approach described here will be complemented by these developments progressively improving accuracy and applicability.

The APT-based methods developed in this study represent a bridge between microstructure and micro-property. This outlook opens the pathway for a formidable nexus between the determination of the structure of a material and its properties by using a range of other atomistic simulations (such as MC and MD), particularly for intrinsically highly inhomogeneous systems. One could also conceive of large-scale orbitalfree DFT ${ }^{39}$ so as to relax the hybrid atom probe data and circumvent the limitation currently associated with the hard lattice originating from the rectification procedure, which could be of paramount interest for replacing interstitial atoms or obtain the appropriate local crystal structure of precipitates, for example, allowing for comparison with other characterization techniques such as electron microscopy or X-ray or neutron scattering.

\section{Methods}

Atom probe experiments. $\mathrm{Al}-5.6 \mathrm{Ag}-0.84 \mathrm{Cu}$ (at. \%) alloy samples were solution treated for $1 \mathrm{~h}$ at $525^{\circ} \mathrm{C}$ in a salt bath and cold water $\left(15-20^{\circ} \mathrm{C}\right)$ quenched. Specimens for APT were prepared first by cutting small blanks $(0.3 \mathrm{~mm} \times 0.3$ $\mathrm{mm} \times 15 \mathrm{~mm}$ ) from the bulk alloy material before thermal ageing. Needle-shaped specimens for APT analysis, with radii $\sim 50 \mathrm{~nm}$, were prepared using standard two-stage electropolishing techniques ${ }^{4}$.

APT analysis was performed using a local electrode atom probe $3000 \mathrm{X} \mathrm{Si}$ manufactured by Cameca. Experiments were performed at a specimen temperature of $\sim 20-25 \mathrm{~K}$ under ultrahigh vacuum conditions of $\sim 1 \times 10^{-8} \mathrm{~Pa}$, in voltagepulsing mode with a pulse fraction of $20 \%$ and a pulse repetition rate of $200 \mathrm{kHz}$. Reconstruction of the APT data was performed using the IVAS software package, which is based on a reverse-projection algorithm ${ }^{40}$, and was calibrated using techniques developed by Gault et al. ${ }^{41,42}$
SRO-based MC simulations. The first step in our process after acquiring and reconstructing the atom probe data is to characterize the average local chemical distribution via the measurement of SRO parameters. The GM-SRO ${ }^{22}$ is based on the probability of finding particular types of atoms around another atom defined as

$$
p_{\left\{B_{j}\right\}_{j=1}^{k}\left\{B_{l}\right\}_{l=0}^{h}}^{m}=p_{\left\{B_{1}|\ldots| B_{k}\right\}\left\{B_{1}|\ldots| B_{h}\right\}}^{m}=\frac{\sum_{j=1}^{k} N_{B_{j}} \sum_{l=h}^{u} p_{B_{j} B_{l}}^{m}}{\sum_{j=1}^{k} N_{B_{j}}} .
$$

Taking the example of an $\mathrm{Al}-\mathrm{Ag}-\mathrm{Cu}$ alloy, $p_{\{\mathrm{Ag}\}\{\mathrm{Cu}\}}^{m=1}$ represents the probability of finding a $\mathrm{Cu}$ atom in the first shell $(m=1)$ around an $\mathrm{Ag}$ atom. Likewise, $p_{\{\mathrm{Cu}\}\{\mathrm{Al}, \mathrm{Cu}\}}^{m}$ represents the probability of finding an $\mathrm{Ag}$ or $\mathrm{Cu}$ atom in the third crystallographic shell around $\mathrm{Cu}$ atom. The resulting GM-SRO expression is given by

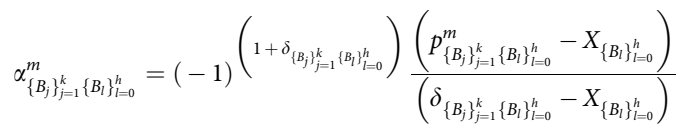

where $\delta_{\left\{B_{j}\right\}_{j=1}^{k}\left\{B_{l}\right\}_{l=0}^{h}}$ equals 1 if the two sets contain exactly the same atomic species and 0 otherwise, and $X_{\left\{B_{j}\right\}_{j=1}^{k}}=X_{B_{1}}+\ldots+X_{B_{k}}$. Again taking the current example, this results in a set of possible parameters

$$
\boldsymbol{\alpha}=\left(\alpha_{\{\mathrm{Al}\}\{\mathrm{Ag}\}}^{m}, \alpha_{\{\mathrm{Al}\}\{\mathrm{Cu}\}}^{m}, \alpha_{\{\mathrm{Al}\}\{\mathrm{Al}\}}^{m}, \alpha_{\{\mathrm{Ag}\}\{\mathrm{Al}\}}^{m}, \ldots, \alpha_{\{\mathrm{AlAgCu}\}\{\mathrm{AgCu}\}}^{m}\right)^{m=1,2,3, \ldots}
$$

where each element of this set can be measured as a function of radius, $m$. This represents a comprehensive statistical description of the system. An approach for measuring GM-SRO in APT reconstructions, taking into account its finite spatial resolution and limited detected efficiency, has previously been developed ${ }^{22}$.

It has previously been demonstrated that in many cases, the SRO contains sufficient information to accurately predict the identities of the atoms missing due to finite detection in an APT experiment ${ }^{28}$. MC approaches have previously been developed to drive a simulated atomic system to a specific configuration based on using experimentally measured SRO as target parameter ${ }^{28,43}$. Starting at some initial configuration atoms in a lattice, in each step of an SRO-based MC simulation, the relative positions of two atoms are swapped at random. After each trial move, the set of SRO is re-evaluated and if the swap drives these parameters towards their target values the move is accepted, otherwise it is rejected and the atoms are replaced to their original positions. Defining whether or not the swapping of a pair of atoms has improved the overall configuration of the system is not straightforward. The target SRO can potentially comprise a large set of parameters. Taking the example above, it is possible that the action of randomly swapping a pair of atoms may move the system towards the $\alpha_{\{\mathrm{Ag}\}\{\mathrm{Cu}\}}^{m}$ target but simultaneously also move the system away from the $\alpha_{\{\mathrm{Cu}\}\{\mathrm{Cu}\}}^{m}$ target. Hence, an objective metric is required in the algorithm to decide on whether the trial move has either improved or worsened the overall atomic configuration. Here, we define residual as a measure of the difference in the instantaneous SRO in the simulated system and the experimentally measured target SRO values (denoted by the prefix $T$ ):

$$
\Delta_{\mathrm{SRO}}=\sum\left|\alpha_{\{i\}\{j\}}^{m}-{ }^{T} \alpha_{\{i\}\{j\}}^{m}\right|
$$

A swap reducing the value of $\Delta_{\text {SRO }}$ is automatically accepted. The required number of trial moves is dependent on the magnitude of the difference between the target and the current actual SRO parameter values, the concentration of each element, number of crystallographic shells, size of the data set and the number of elements that constitute the alloy.

Lattice rectification. In APT, crystallographic information is most apparent in the form of crystallographic planes. Spatial distribution maps provide an efficient approach for the characterization of these atomic planes (Supplementary Fig. 2), in terms of inter-planar spacing, orientation and resolution (that is, the accuracy to which atoms are placed on these planes). Knowledge of this underlying lattice structure combined with the local distribution of atoms in the raw data facilitates the remapping of the perturbed atom positions back to their true lattice sites, a process that we termed lattice rectification ${ }^{26}$.

Having identified the orientation of a set of crystallographic planes $\{h k l\}$, it can be inferred that in the specimen, any two neighbouring atoms were actually separated by a distance corresponding to an exact multiple of the plane spacing in this direction. A small correction can be applied to the reconstructed coordinates of atoms to satisfy this condition. If three independent sets of planes are identified within the data, this correction of atoms onto the closest plane, simultaneously in three directions, provides a unique solution, that is, a single lattice site. The significance of this result is that the lattice structure of the specimen is recovered via a progressive atom-by-atom triangulation.

The smallest possible correction is being made to the atomic coordinates, requiring access to highly spatially resolved data. However, trajectory aberrations can result in significant inaccuracies in the original 3D positioning of an atom. Trajectory aberrations most often originate primarily from local magnification effects on the specimen surface arising from either the preferential evaporation or 
retention of certain atoms. Such aberrations cause two main issues: (1) the crystalline lattice appears smeared out by small dithering of the atomic positions and (2) the shape of larger features (that is, precipitate, layers and so on) appears distorted. These effects have been thoroughly described in the literature, including in recent reviews on the topic ${ }^{44-46}$. However, as shown in this study, it is often possible to identify high-quality subvolumes of the data devoid of significant local magnification effects. This simple lattice rectification approach requires APT reconstructions within which sufficient crystallographic information can be identified, and incorporate significant regions of interest free of aberrations. It is noted that this is currently not the case for many complex systems now studied by APT. Although some of these contain a significant amount of crystallographic information ${ }^{4}$, until the accuracy the original reconstruction can be improved, lattice rectification is not meaningful for these systems.

DFT simulations. All-electron DFT calculations were performed using the generalized gradient approximation ${ }^{48}$ with the DMol3 program package ${ }^{49}$. The wave functions were expanded in terms of a double-numerical quality basis set, with a set of large values of real-space cut-off $(\mathrm{Cu}$ : $9.45 \mathrm{Bohr}$; Ag: $9.98 \mathrm{Bohr}$; Al: $10.88 \mathrm{Bohr})$. A reciprocal space of $4 \times 4 \times 4 \mathrm{~K}$-point meshes was employed in the calculations of the 108 -atom supercells.

\section{References}

1. Nellist, P. D. et al. Direct sub-angstrom imaging of a crystal lattice. Science 305, 1741-1741 (2004)

2. Muller, D. A. et al. Atomic-scale chemical imaging of composition and bonding by aberration-corrected microscopy. Science 319, 1073-1076 (2008).

3. Midgley, P. A. \& Dunin-Borkowski, R. E. Electron tomography and holography in materials science. Nat. Mater. 8, 271-280 (2009).

4. Miller, M. K., Cerezo, A., Hetherington, M. G. \& Smith, G. D. W. Atom Probe Field Ion Microscopy (Oxford Science Publications - Clarendon Press, 1996).

5. Kelly, T. F. \& Miller, M. K. Invited review article: atom probe tomography. Rev. Sci. Instrum. 78, 031101 (2007).

6. Seidman, D. N. Three-dimensional atom-probe tomography: advances and applications. Annu. Rev. Mat. Res. 37, 127-158 (2007).

7. Vitek, V. \& Srolovitz, D. J. Atomistic Simulation of Materials: Beyond Pair Potentials (Plenum Press, 1989).

8. Gross, D. \& Li, M. Constructing microstructures of poly- and nanocrystalline materials for numerical modeling and simulation. Appl. Phys. Lett. 80, 746-748 (2002).

9. Mao, Z. G., Sudbrack, C. K., Yoon, K. E., Martin, G. \& Seidman, D. N. The mechanism of morphogenesis in a phase-separating concentrated multicomponent alloy. Nat. Mater. 6, 210-216 (2007).

10. Pareige, C., Soisson, F., Martin, G. \& Blavette, D. Ordering and phase separation in Ni-Cr-Al: Monte Carlo simulations vs three-dimensional atom probe. Acta Mater. 47, 1889-1899 (1999).

11. Sha, G. \& Cerezo, A. Kinetic Monte Carlo simulation of clustering in an Al-Zn-Mg-Cu alloy(7050). Acta Mater. 53, 907-917 (2005).

12. Clouet, E. et al. Complex precipitation pathways in multicomponent alloys. Nat. Mater. 5, 482-488 (2006).

13. Zhou, X. W. et al. Atomic scale structure of sputtered metal multilayers. Acta Mater. 49, 4005-4015 (2001).

14. Balogh, Z., Chellali, M. R., Greiwe, G. H., Schmitz, G. \& Erdelyi, Z. Interface sharpening in miscible $\mathrm{Ni} / \mathrm{Cu}$ multilayers studied by atom probe tomography. Appl. Phys. Lett. 99, 181902 (2011).

15. Amouyal, Y., Mao, Z. \& Seidman, D. N. Phase partitioning and site-preference of hafnium in the gamma '(L1(2))/gamma(fcc) system in Ni-based superalloys: an atom-probe tomographic and first-principles study. Appl. Phys. Lett. 95, 161909 (2009)

16. Gault, B. et al. Atom probe tomography investigation of $\mathrm{Mg}$ site occupancy within delta' precipitates in an Al-Mg-Li alloy. Scr. Mater. 66, 903-906 (2012).

17. Geng, W. T., Ping, D. H., Gu, Y. F., Cui, C. Y. \& Harada, H. Stability of nanoscale co-precipitates in a superalloy: a combined first-principles and atom probe tomography study. Phys. Rev. B 76, 224102 (2007).

18. Li, L. et al. Magnetism of Co-doped $\mathrm{ZnO}$ epitaxially grown on a $\mathrm{ZnO}$ substrate. Phys. Rev. B 85, 174430 (2012).

19. Yeoh, W. K. et al. Direct observation of local potassium variation and its correlation to electronic inhomogeneity in (Ba1-xKx)Fe2As2 pnictide. Phys. Rev. Lett. 106, 247002 (2011).

20. Cadel, E., Vurpillot, F., Larde, R., Duguay, S. \& Deconihout, B. Depth resolution function of the laser assisted tomographic atom probe in the investigation of semiconductors. J. Appl. Phys. 106, 1-6 (2009).

21. Gault, B. et al. Origin of the spatial resolution in atom probe microscopy. Appl. Phys. Lett. 95, 034103 (2009).

22. Ceguerra, A. V., Powles, R. C., Moody, M. P. \& Ringer, S. P. Quantitative description of atomic architecture in solid solutions: a generalized theory for multicomponent short-range order. Phys. Rev. B 82, 132201 (2010).

23. Geiser, B. P., Kelly, T. F., Larson, D. J., Schneir, J. \& Roberts, J. P. Spatial distribution maps for atom probe tomography. Microsc. Microanal. 13, 437-447 (2007)
24. Vurpillot, F., De Geuser, F., Da Costa, G. \& Blavette, D. Application of Fourier transform and autocorrelation to cluster identification in the three-dimensional atom probe. J. Microsc. 216, 234-240 (2004).

25. Yao, L. et al. Crystallographic structural analysis in atom probe microscopy via 3D Hough transformation. Ultramicroscopy 111, 458-463 (2011).

26. Moody, M. P. et al. Lattice rectification in atom probe tomography: toward true three-dimensional atomic microscopy. Microsc. Microanal. 17, 226-239 (2011).

27. Vurpillot, F., Renaud, L. \& Blavette, D. A new step towards the lattice reconstruction in 3DAP. Ultramicroscopy 95, 223-230 (2003).

28. Ceguerra, A. V. et al. Short-range order in multicomponent materials. Acta Crystallogr. A 68, 547-560 (2012).

29. Moody, M. P., Gault, B., Stephenson, L. T., Haley, D. \& Ringer, S. P. Qualification of the tomographic reconstruction in atom probe by advanced spatial distribution map techniques. Ultramicroscopy 109, 815-824 (2009).

30. Danoix, F., Miller, M. K. \& Bigot, A. Analysis conditions of an industrial $\mathrm{Al}-\mathrm{Mg}-\mathrm{Si}$ alloy by conventional and $3 \mathrm{D}$ atom probes. Ultramicroscopy $\mathbf{8 9}$, 177-188 (2001).

31. Saxey, D. W. Correlated ion analysis and the interpretation of atom probe mass spectra. Ultramicroscopy 111, 473-479 (2011).

32. Turchanin, M. A. \& Agraval, P. G. Cohesive energy, properties, and formation energy of transition metal alloys. Powder Metall. Met. Ceram. 47, 26-39 (2008).

33. Ashcroft, N. W. \& Mermin, N. D. Solid State Physics (Saunders College, 1976)

34. Kittel, C. Introduction to Solid State Physics (Wiley, 2004).

35. Born, M. \& Huang, K. Dynamical Theory of Crystal Lattices (Clarendon Press, 1956).

36. Fulcher, B. D., Cui, X. Y., Delley, B. \& Stampfl, C. Hardness analysis of cubic metal mononitrides from first principles. Phys. Rev. B 85, 184106 (2012).

37. Gavini, V., Bhattacharya, K. \& Ortiz, M. Quasi-continuum orbital-free densityfunctional theory: a route to multi-million atom non-periodic DFT calculation. J. Mech. Phys. Solids 55, 697-718 (2007).

38. Gustafsson, A., Pistol, M.-E., Montelius, L. \& Samuelson, L. Local probe techniques for luminescence studies of low-dimensional semiconductor structures. J. Appl. Phys. 84, 1715-1775 (1998).

39. Storm, K. et al. Spatially resolved Hall effect measurement in a single semiconductor nanowire. Nat. Nanotechnol. 7, 718-722 (2012).

40. Gault, B. et al. Advances in the reconstruction of atom probe tomography data. Ultramicroscopy 111, 448-457 (2011).

41. Gault, B. et al. Estimation of the reconstruction parameters for atom probe tomography. Microsc. Microanal. 14, 296-305 (2008).

42. Gault, B. et al. Advances in the calibration of atom probe tomographic reconstruction. art. no. 034913. J. Appl. Phys. 105, 34913 (2009).

43. de Fontaine, D. The number of independent pair-correlation functions in multicomponent systems. J. Appl. Crystallogr. 4, 15-19 (1971).

44. Miller, M. K. \& Hetherington, M. G. Local magnification effects in the atom probe. Surf. Sci. 246, 442-449 (1991).

45. Vurpillot, F., Cerezo, A., Blavette, D. \& Larson, D. J. Modeling image distortions in 3DAP. Microsc. Microanal. 10, 384-390 (2004).

46. Larson, D. J., Gault, B., Geiser, B. P., De Geuser, F. \& Vurpillot, F. Atom probe tomography spatial reconstruction: status and directions. Curr. Opin. Solid State Mater. Sci. 17, 236-247 (2013)

47. Gault, B., Moody, M. P., Cairney, J. M. \& Ringer, S. P. Atom probe crystallography. Mater. Today 15, 378-386 (2012).

48. Perdew, J. P., Burke, K. \& Ernzerhof, M. Generalized gradient approximation made simple. Phys. Rev. Lett. 77, 3865-3868 (1996).

49. Delley, B. From molecules to solids with the DMol(3) approach. J. Chem. Phys. 113, 7756-7764 (2000)

\section{Acknowledgements}

We are grateful for scientific and technical input and support from staff and students from the Department of Materials at the University of Oxford and the Australian Microscopy \& Microanalysis Research Facility (AMMRF) at The University of Sydney. We acknowledge the computing resources provided by the National Computational Infrastructure (Australia) and support from the Australian Research Council.

\section{Author contributions}

M.P.M. and S.P.R. led the research. M.P.M. developed the lattice rectification algorithm and contributed to development of the SRO measurements and the SRO-based MC simulations. A.V.C. designed the SRO measurements and the SRO-based MC simulations, and contributed to the design of the DFT simulations. A.J.B. contributed to the development of the lattice rectification methodology and the MC simulations. X.Y.C. designed, undertook and interpreted the DFT simulations. B.G. generated the simulated APT-like data, contributed to the development of the analytical techniques and the application of the data in DFT simulations. L.T.S. and R.K.W.M. undertook the APT experiments, provided the reconstructions and contributed to development of the analytical techniques. R.C.P. contributed to the development of the SRO measurements and the SRO-based MC simulations. S.P.R. directed the research programme. All authors discussed results and contributed to writing of the paper. 


\section{Additional information}

Supplementary Information accompanies this paper at http://www.nature.com/ naturecommunications

Competing financial interests: The authors declare no competing financial interests.
Reprints and permission information is available online at http://www.nature.com/ reprintsandpermissions/

How to cite this article: Moody, M. P. et al. Atomically resolved tomography to directly inform simulations for structure-property relationships. Nat. Commun. 5:5501 doi: 10.1038/ncomms6501 (2014). 